\title{
A Simplex Sufficiency Condition for Quadrature Formulas
}

\author{
By C. S. Duris
}

1. Introduction. In this paper a procedure is described for deriving interpolatory type quadrature formulas by inverting linear systems of differentiation formulas. Because interpolatory type quadrature formulas are uniquely given in terms of the interval of integration and the interpolating points (see Krylov [6]), no new integration formulas result. By carrying along the remainder terms for the differentiation formulas, remainder terms for the quadrature formulas can be obtained. Using this procedure a sufficient condition is given for a quadrature formula to have a remainder of the form $M f^{(n)}(\xi)$, where $f$ is the function being integrated and $M$ is a constant. This is equivalent to the statement that the formula is simplex (see Daniell [8]). Applications of this sufficiency condition are made, and in particular a fairly thorough investigation of the formulas having form

$$
\int_{-1}^{1} f(t) d t \sim C_{0} f(-1)+C_{1} f(\alpha)+C_{2} f(\beta)+C_{3} f(1)
$$

is made for $-1<\alpha>,<1$.

2. Numerical Differentiation Formulas. Let $y(x) \in C^{n+1}[a, b]$. The Lagrange interpolation formula with remainder, which interpolates $y(x)$ at the $n+1$ distinct points $x_{0}, x_{1}, \cdots, x_{n} \in[a, b]$ is given by (see [1], [2], [3], [6], or [7])

$$
y(x)=\sum_{j=0}^{n} l_{j}^{n}(x) y_{j}+E_{n}(y ; x),
$$

where $y_{j}=y\left(x_{j}\right)$ and

$$
l_{j}^{n}(x)=\prod_{k=0 ; k \neq j}^{n} \frac{\left(x-x_{k}\right)}{\left(x_{j}-x_{k}\right)} .
$$

The remainder term $E_{n}(y ; x)$ can be expressed in terms of a divided difference as (using notation of [1])

$$
E_{n}(y ; x)=\pi_{n}(x) y\left[x_{0}, x_{1}, \cdots, x_{n}, x\right],
$$

where $\pi_{n}(x)=\left(x-x_{0}\right)\left(x-x_{1}\right) \cdots\left(x-x_{n}\right)$, or in terms of an $(n+1)$ st derivative as

$$
E_{n}(y ; x)=\pi_{n}(x) \frac{y^{(n+1)}(\xi(x))}{(n+1) !},
$$

where $\min \left(x_{0}, \cdots, x_{n}, x\right)<\xi(x)<\max \left(x_{0}, \cdots, x_{n}, x\right)$. In most of the analysis we will choose to use the remainder term as it appears in (2.3).

In the following $P_{n}$ is the linear space of dimension $n+1$ consisting of all real

Received April 20, 1965. 
polynomials of degree less than or equal to $n$. An immediate consequence of the Lagrange interpolation formula is

Lemma 2.1. The $n$th degree polynomials $l_{k}{ }^{n}(x)$ for $k=0,1, \cdots, n$ given by $(2.2)$ form a basis for $P_{n}$.

Using the notation

$$
d_{j}^{n}(x)=\frac{d}{d x} l_{j}^{n}(x)
$$

and differentiating (2.1) we get

$$
y^{\prime}(x)=\sum_{j=0}^{n} d_{j}^{n}(x) y_{j}+R_{n}^{\prime}(x)
$$

where

$$
R_{n}^{\prime}(x)=\pi_{n}{ }^{\prime}(x) y\left[x_{0}, \cdots, x_{n}, x\right]+\pi_{n}(x) y\left[x_{0}, \cdots, x_{n}, x, x\right] .
$$

A second lemma of importance is now given.

Lemma 2.2. The $(n-1)$ st degree polynomials $d_{j}{ }^{n}(x)$ for $j=0,1, \cdots, n$ span the space $P_{n-1}$, and any $n$ of these $n+1$ polynomials form a basis for $P_{n-1}$.

Proof. Every polynomial $q \in P_{n-1}$ is the derivative of a polynomial $p \in P_{n}$. Thus for any $q \in P_{n-1}$

$$
q(x)=p^{\prime}(x)=d_{0}{ }^{n}(x) p\left(x_{0}\right)+d_{1}{ }^{n}(x) p\left(x_{1}\right)+\cdots+d_{n}{ }^{n}(x) p\left(x_{n}\right) .
$$

Hence $d_{j}^{n}(x)$ for $j=0,1, \cdots, n \operatorname{span} P_{n-1}$. Since $1 \in P_{n}$ we know that

$$
d_{0}{ }^{n}(x)+d_{1}{ }^{n}(x)+\cdots+d_{n}{ }^{n}(x)=0 .
$$

Thus $d_{k}{ }^{n}(x)=-\sum_{j=0 ; j \neq k}^{n} d_{j}{ }^{n}(x)$, which implies that any set $d_{0}{ }^{n}(x), \cdots, d_{i-1}^{n}(x)$, $d_{i+1}^{n}(x), \cdots, d_{n}{ }^{n}(x)$ is a basis for $P_{n-1}$. This proves Lemma 2.2.

Let $z_{0}, z_{1}, \cdots, z_{n}$ be $n+1$ distinct points in $[a, b]$ not necessarily equal to the $x_{i}$ 's defining the $l_{j}^{n}(x)$ 's. If we evaluate (2.6) at these $z_{i}$ 's we get the following system of $n+1$ equations:

$$
\begin{aligned}
& y^{\prime}\left(z_{0}\right)=d_{0}{ }^{n}\left(z_{0}\right) y_{0}+d_{1}{ }^{n}\left(z_{0}\right) y_{1}+\cdots+d_{n}{ }^{n}\left(z_{0}\right) y_{n}+R_{n}{ }^{\prime}\left(z_{0}\right) \\
& y^{\prime}\left(z_{1}\right)=d_{0}{ }^{n}\left(z_{1}\right) y_{0}+d_{1}{ }^{n}\left(z_{1}\right) y_{1}+\cdots+d_{n}{ }^{n}\left(z_{1}\right) y_{n}+R_{n}{ }^{\prime}\left(z_{1}\right) \\
& y^{\prime}\left(z_{n}\right)=d_{0}{ }^{n}\left(z_{n}\right) y_{0}+d_{1}{ }^{n}\left(z_{n}\right) y_{1}+\cdots+d_{n}{ }^{n}\left(z_{n}\right) y_{n}+R_{n}{ }^{\prime}\left(z_{n}\right) .
\end{aligned}
$$

Recall that $y_{k}=y\left(x_{k}\right)$.

The $(n+1) \times(n+1)$ matrix

$$
W=\left(\begin{array}{cccc}
d_{0}{ }^{n}\left(z_{0}\right) & d_{1}{ }^{n}\left(z_{0}\right) & \cdots & d_{n}{ }^{n}\left(z_{0}\right) \\
d_{0}{ }^{n}\left(z_{1}\right) & d_{1}{ }^{n}\left(z_{1}\right) & \cdots & d_{n}{ }^{n}\left(z_{1}\right) \\
\vdots & \vdots & & \vdots \\
d_{0}{ }^{n}\left(z_{n}\right) & d_{1}^{n}\left(z_{n}\right) & \cdots & d_{n}{ }^{n}\left(z_{n}\right)
\end{array}\right)
$$


is the matrix representation of the operator $d / d x$ on $P_{n}$ with respect to the basis

$$
L_{k}{ }^{n}(z)=\prod_{j=0 ; j \neq k}^{n}\left(\frac{z-z_{k}}{z_{j}-z_{k}}\right) \quad \text { for } k=0,1, \cdots, n .
$$

Thus as a corollary to Lemma 2.2 we have

Corollary 2.2. The rank of $W$ is $n$ and every $n \times n$ submatrix of $W$ is nonsingular.

Proof. It is quite clear from Lemma 2.2 that the rank of $W$ is $n$. Consider now for $0 \leqq i, j \leqq n$ the submatrix

$$
\left(\begin{array}{cccccc}
d_{0}{ }^{n}\left(z_{0}\right) & \cdots & d_{j-1}^{n}\left(z_{0}\right) & d_{j+1}^{n}\left(z_{0}\right) & \cdots & d_{n}{ }^{n}\left(z_{0}\right) \\
\vdots & & \vdots & \vdots & & \vdots \\
d_{0}{ }^{n}\left(z_{i-1}\right) & \cdots & d_{j-1}^{n}\left(z_{i-1}\right) & d_{j+1}^{n}\left(z_{i-1}\right) & \cdots & d_{n}{ }^{n}\left(z_{i-1}\right) \\
d_{0}{ }^{n}\left(z_{i+1}\right) & \cdots & d_{j-1}^{n}\left(z_{i+1}\right) & d_{j+1}^{n}\left(z_{i+1}\right) & \cdots & d_{n}{ }^{n}\left(z_{i+1}\right) \\
\vdots & & \vdots & \vdots & & \vdots \\
d_{0}^{n}\left(z_{n}\right) & \cdots & d_{j-1}^{n}\left(z_{n}\right) & d_{j+1}^{n}\left(z_{n}\right) & \cdots & d_{n}^{n}\left(z_{n}\right)
\end{array}\right) .
$$

The columns of this matrix are the components of $d_{k}{ }^{n}(z)$ with respect to a basis of Lagrange coefficients using $z_{0}, \cdots, z_{i-1}, z_{i+1}, \cdots, z_{n}$ for interpolating points. Thus by Lemma 2.2 these columns are linearly independent and hence the matrix is nonsingular. This proves the corollary.

3. Derivation of Quadrature Formulas. If we let $y(x)=\int_{x_{0}}^{x} f(t) d t$ and we drop the first equation in (2.8) we get the system of equations

$$
\left(\begin{array}{cccc}
d_{11} & d_{12} & \cdots & d_{1 n} \\
d_{21} & d_{22} & \cdots & d_{2 n} \\
\vdots & & & \\
d_{n 1} & d_{n 2} & \cdots & d_{n n}
\end{array}\right)\left(\begin{array}{l}
\int_{x_{0}}^{x_{1}} f(x) d x \\
\int_{x_{0}}^{x_{2}} f(x) d x \\
\vdots \\
\int_{x_{0}}^{x_{n}} f(x) d x
\end{array}\right)=\left(\begin{array}{c}
f\left(z_{1}\right)-R_{n}{ }^{\prime}\left(z_{1}\right) \\
f\left(z_{2}\right)-R_{n}{ }^{\prime}\left(z_{2}\right) \\
\vdots \\
f\left(z_{n}\right)-R_{n}{ }^{\prime}\left(z_{n}\right)
\end{array}\right) .
$$

Here we have used the notation $d_{i j}=d_{j}{ }^{n}\left(z_{i}\right)$ for $1 \leqq i, j \leqq n$.

Let $D=\left(d_{i j}\right)$ be the $n \times n$ matrix in (3.1) and let $D_{i j}$ be the $(i, j)$ th cofactor of the matrix $D$. Since by Corollary 2.2 we know $D$ is nonsingular we get the following theorem by applying Cramer's Rule to (3.1).

TheOREM 3.1. The $n \times n$ system of equations (3.1) can be solved to obtain the integration formulas

$$
\int_{x_{0}}^{x_{k}} f(x) d x=\sum_{i=1}^{n} C_{i k}^{n} f\left(z_{i}\right)+R_{n}\left(f ; x_{0}, x_{k}\right)
$$

for $k=1,2, \cdots, n$ where

$$
C_{i k}^{n}=\frac{D_{i k}}{\operatorname{det}(D)}
$$


and

$$
R_{n}\left(f ; x_{0}, x_{k}\right)=-\sum_{i=1}^{n} C_{i k}^{n} R_{n}^{\prime}\left(z_{i}\right)
$$

The term $R_{n}\left(f ; x_{0}, x_{k}\right)$ is the remainder term for the integration formula. It is interesting to note that we obtain the remainder for this integration formula by applying the integration formula to the negative of the remainder term for differentiation.

A formula is said to be exact for a specified function if the remainder term is zero for that function.

Lemma 3.2. Formula (3.2) is exact when $f$ is a polynomial of degree $n-1$ or less.

Proof. Since the differentiation formulas in (2.8) are exact when $y(x)$ is a polynomial of degree $n$ or less, the integration formula (3.2) obtained from (3.1) will be exact when $f(x)$ is a polynomial of degree $n-1$ or less.

Lemma 3.3. The coefficients $C_{j k}^{n}$ given by (3.3) can also be found by

$$
C_{j k}^{n}=\int_{x_{0}}^{x_{k}} L_{j}{ }^{n-1}(z) d z \quad \text { for } j=1,2, \cdots, n
$$

where

$$
L_{j}^{n-1}(z)=\prod_{i=1 ; i \neq j}^{n} \frac{\left(z-z_{i}\right)}{\left(z_{j}-z_{i}\right)} .
$$

Proof. Since (3.2) is exact for polynomials of degree $n-1$ or less we may put $f(z)=L_{j}{ }^{n-1}(z)$ and know that

$$
\sum_{i=1}^{n} C_{i k}^{n} L_{j}^{n-1}\left(z_{i}\right)=\int_{x_{0}}^{x_{k}} L_{j}^{n-1}(z) d z .
$$

Noting that $L_{j}{ }^{n-1}\left(z_{i}\right)=0$ if $j \neq i$ and $L_{j}{ }^{n-1}\left(z_{j}\right)=1$ we get (3.5). This proves the desired lemma.

From Lemma 3.3 we learn that formula (3.2) does not really depend on any of the $x_{i}$ 's forming the system of equations (3.1) except $x_{0}$ and $x_{k}$. For this reason we will take $x_{i}=z_{i+1}$ for $1 \leqq i \leqq n-1$. Note tinat if the upper limit, $x_{k}$, of the integration is not one of the $z_{i}$ 's we may take $k=n$, because $x_{n}$ is not restricted to be one of the $z_{i}$ 's.

Hence if $x_{i}=z_{i+1}$ for $1 \leqq i \leqq n-1$ term $\pi_{n}(x)$ in (2.3) becomes

$$
\pi_{n}(x)=\left(x-x_{0}\right)\left(x-z_{2}\right) \cdots\left(x-z_{n}\right)\left(x-x_{n}\right) .
$$

For convenience in what is to follow we will write $\pi_{n}(x)=\pi_{n-1}(x)\left(x-x_{n}\right)$, where

$$
\pi_{n-1}(x)=\left(x-x_{0}\right)\left(x-z_{2}\right) \cdots\left(x-z_{n}\right) .
$$

Thus the remainder terms for differentiation at the $z_{i}$ 's are

$$
R_{n}^{\prime}\left(z_{i}\right)=\pi_{n-1}^{\prime}\left(z_{i}\right)\left(z_{i}-x_{n}\right) y\left[x_{0}, z_{2}, \cdots, z_{n}, x_{n}, z_{i}\right]
$$


for $2 \leqq i \leqq n$ and

$$
\begin{aligned}
R_{n}{ }^{\prime}\left(z_{1}\right)= & \pi_{n}{ }^{\prime}\left(z_{1}\right) y\left[x_{0}, z_{2}, \cdots, z_{n}, x_{n}, z_{1}\right] \\
& +\pi_{n}\left(z_{1}\right) y\left[x_{0}, z_{2}, \cdots, z_{n}, x_{n}, z_{1}, z_{1}\right] .
\end{aligned}
$$

For our purposes we will modify (3.11). Note that

$$
\begin{aligned}
& \pi_{n}\left(z_{1}\right) y\left[x_{0}, z_{2}, \cdots, z_{n}, x_{n}, z_{1}, z_{1}\right] \\
& -\pi_{n-1}\left(z_{1}\right)\left\{y\left[x_{0}, z_{2}, \cdots, z_{n}, z_{1}, z_{1}\right]-y\left[x_{0}, z_{2}, \cdots, z_{n}, x_{n}, z_{1}\right]\right\},
\end{aligned}
$$

and $\pi_{n}^{\prime}\left(z_{1}\right)=\pi_{n-1}^{\prime}\left(z_{1}\right)\left(z_{1}-x_{n}\right)+\pi_{n-1}\left(z_{1}\right)$. Hence (3.11) can be written as

$$
\begin{aligned}
R_{n}{ }^{\prime}\left(z_{1}\right)= & \pi_{n-1}^{\prime}\left(z_{1}\right)\left(z_{1}-x_{n}\right) y\left[x_{0}, z_{2}, \cdots, z_{n}, x_{n}, z_{1}\right] \\
& +\pi_{n-1}\left(z_{1}\right) y\left[x_{0}, z_{2}, \cdots, z_{n}, z_{1}, z_{1}\right] .
\end{aligned}
$$

Using (3.10) and (3.13) in (3.4) we get

$$
\begin{aligned}
R_{n}\left(f ; x_{0}, x_{k}\right)= & -\sum_{i=1}^{n} C_{i k}^{n} \pi_{n-1}^{\prime}\left(z_{i}\right)\left(z_{i}-x_{n}\right) y\left[x_{0}, z_{2}, \cdots, z_{n}, x_{n}, z_{i}\right] \\
& -C_{1 k}^{n} \pi_{n-1}\left(z_{1}\right) y\left[x_{0}, z_{2}, \cdots, z_{n}, z_{1}, z_{1}\right] .
\end{aligned}
$$

4. A Simplex Sufficiency Condition. In [8] Daniell studies remainders for interpolation and quadrature formulas. In this paper he defined simplex formulas. This definition is (see also Kunz [7]):

Definition 4.1. A formula is said to be simplex of order $m$, if

(1) The formula is exact for polynomials of degree $m-1$ or less and not exact for $x^{m}$.

(2) If the formula is exact for any function $f \in C^{m}[a, b]$, then $f^{(m)}(x)=0$ for some $x \in[a, b]$.

From [7] or [8] we learn the following about the formulas given by (3.2):

LEMMA 4.1. The integration formula

$$
\int_{x_{0}}^{x_{k}} f(x) d x=\sum_{i=1}^{n} C_{i k}^{n} f\left(z_{i}\right)+R_{n}\left(f ; x_{0}, x_{k}\right)
$$

is simplex of order $n$ if and only if

$$
R_{n}\left(f ; x_{0}, x_{k}\right)=M f^{(n)}(\xi)
$$

where $\xi$ is some point in $[a, b]$ and $M$ is a nonzero constant.

Since $d^{n} x^{n} / d x^{n}=n$ !, Daniell [8] shows that in (4.1)

$$
M=\frac{1}{n !}\left\{\int_{x_{0}}^{x_{k}} x^{n} d x-\sum_{i=1}^{n} C_{i k}^{n} z_{i}^{n}\right\} .
$$

We are now in position to prove the following theorem which gives a sufficient condition for formulas of type (3.2) to be simplex. For convenience in stating this theorem we have defined $z_{n+1}$ to be $x_{n}$ in (3.14). Recall also that in (3.14)

$$
\pi_{n-1}(x)=\left(x-x_{0}\right)\left(x-z_{2}\right) \cdots\left(x-z_{n}\right) .
$$


Theorem 4.2. If for $s=1,2, \cdots, n$ the $n$ quantities

$$
\left(z_{s}-z_{s+1}\right) \sum_{i=1}^{s} C_{i k}^{n} \pi_{n-1}^{\prime}\left(z_{i}\right)
$$

have the same sign, and $C_{1 k}^{n} \pi_{n-1}\left(z_{1}\right)$ is zero or has this same sign, then

$$
R_{n}\left(f ; x_{0}, x_{k}\right)=-y\left[x_{0}, z_{2}, \cdots, z_{n}, \xi, \eta\right] \sum_{i=1}^{n} C_{i k}^{n} \pi_{n}{ }^{\prime}\left(z_{i}\right)
$$

where $\pi_{n}(x)=\pi_{n-1}(x)\left(x-z_{n+1}\right), z_{n+1}=x_{n}$, and $\min \left(z_{1}, \cdots, z_{n+1}\right) \leqq \xi$, $\eta \leqq \max \left(z_{1}, \cdots, z_{n+1}\right)$; which implies formula (3.2) is simplex of order $n$.

Proof. Repeating (3.14) here we have

$$
\begin{aligned}
R_{n}\left(f ; x_{0}, x_{k}\right)= & -\sum_{i=1}^{n} C_{i k}^{n} \pi_{n-1}^{\prime}\left(z_{i}\right)\left(z_{i}-z_{n+1}\right) y\left[x_{0}, z_{2}, \cdots, z_{n}, z_{n+1}, z_{i}\right] \\
& -C_{1 k}^{n} \pi_{n-1}\left(z_{1}\right) y\left[x_{0}, z_{2}, \cdots, z_{n}, z_{1}, z_{1}\right] .
\end{aligned}
$$

Considering one term in the summation we have

$$
\begin{aligned}
& C_{i k}^{n} \pi_{n-1}^{\prime}\left(z_{i}\right)\left(z_{i}-z_{n+1}\right) y\left[x_{0}, z_{2}, \cdots, z_{n}, z_{n+1}, z_{i}\right] \\
& \quad=C_{i k \pi}^{n} \pi_{n-1}^{\prime}\left(z_{i}\right)\left\{y\left[x_{0}, z_{2}, \cdots, z_{n}, z_{i}\right]-y\left[x_{0}, z_{2}, \cdots, z_{n}, z_{n+1}\right]\right\} .
\end{aligned}
$$

By telescoping

$$
\begin{aligned}
& y\left[x_{0}, z_{2}, \cdots, z_{n}, z_{i}\right]-y\left[x_{0}, z_{2}, \cdots, z_{n}, z_{n+1}\right] \\
& \quad=\sum_{s=i}^{n}\left\{y\left[x_{0}, z_{2}, \cdots, z_{n}, z_{s}\right]-y\left[x_{0}, z_{2}, \cdots, z_{n}, z_{s+1}\right]\right\} .
\end{aligned}
$$

Now note that

$$
\begin{aligned}
& y\left[x_{0}, z_{2}, \cdots, z_{n}, z_{s}\right]-y\left[x_{0}, z_{2}, \cdots, z_{n}, z_{s+1}\right] \\
& \quad=\left(z_{s}-z_{s+1}\right) y\left[x_{0}, z_{2}, \cdots, z_{n}, z_{s}, z_{s+1}\right] .
\end{aligned}
$$

This gives

$$
\begin{gathered}
y\left[x_{0}, z_{2}, \cdots, z_{n}, z_{i}\right]-y\left[x_{0}, z_{2}, \cdots, z_{n}, z_{n+1}\right] \\
=\sum_{s=i}^{n}\left(z_{s}-z_{s+1}\right) y\left[x_{0}, z_{2}, \cdots, z_{n}, z_{s}, z_{s+1}\right] .
\end{gathered}
$$

Using (4.8) and (4.5) we get

$$
\begin{aligned}
R_{n}\left(f ; x_{0}, x_{k}\right)= & -\sum_{i=1}^{n} C_{i k}^{n} \pi_{n-1}^{\prime}\left(z_{i}\right) \sum_{s=i}^{n}\left(z_{s}-z_{s+1}\right) y\left[x_{0}, z_{2}, \cdots, z_{n}, z_{s}, z_{s+1}\right] \\
& -C_{1 k}^{n} \pi_{n-1}\left(z_{1}\right) y\left[x_{0}, z_{2}, \cdots, z_{n}, z_{1}, z_{1}\right] .
\end{aligned}
$$

We now use the fact that $\sum_{i=1}^{n} a_{i} \sum_{s=i}^{n} b_{s}=\sum_{s=1}^{n} b_{s} \sum_{i=1}^{s} a_{i}$. This gives

$$
\begin{aligned}
R_{n}\left(f ; x_{0}, x_{k}\right) & =-\sum_{s=1}^{n} y\left[x_{0}, z_{2}, \cdots, z_{n}, z_{s}, z_{s+1}\right] \\
& \cdot\left(z_{s}-z_{s+1}\right) \sum_{i=1}^{s} C_{i k}^{n} \pi_{n-1}^{\prime}\left(z_{i}\right)-C_{1 k}^{n} \pi_{n-1}\left(z_{1}\right) y\left[x_{0}, z_{2}, \cdots, z_{n}, z_{1}, z_{1}\right] .
\end{aligned}
$$


Hence if $\left(z_{s}-z_{s+1}\right) \sum_{i=1}^{s} C_{i k}^{n} \pi_{n-1}^{\prime}\left(z_{i}\right)$ do not change sign (some of these terms may be zero) and $C_{1 k}^{n} \pi_{n-1}^{\prime}\left(z_{1}\right)$ has this same sign or is zero

$$
\begin{aligned}
R_{n}\left(f ; x_{0}, x_{k}\right)= & -y\left[x_{0}, z_{2}, \cdots, z_{n}, \xi, \eta\right] \\
& \cdot\left\{\sum_{s=1}^{n}\left(z_{s}-z_{z+1}\right) \sum_{i=1}^{s} C_{i k}^{n} \pi_{n-1}^{\prime}\left(z_{i}\right)+C_{1 k}^{n} \pi_{n-1}\left(z_{1}\right)\right\},
\end{aligned}
$$

where $\min \left(x_{0}, z_{1}, \cdots, z_{n+1}\right) \leqq \xi, \eta \leqq \max \left(x_{0}, z_{1}, \cdots, z_{n+1}\right)$. Noting that

$$
\sum_{s=1}^{n}\left(z_{s}-z_{s+1}\right) \sum_{i=1}^{s} C_{i k}^{n} \pi_{n-1}^{\prime}\left(z_{i}\right)=\sum_{i=1}^{n} C_{i k}^{n} \pi_{n-1}^{\prime}\left(z_{i}\right)\left(z_{i}-z_{n+1}\right)
$$

$x_{k}=z_{k+1}$ for $1 \leqq k \leqq n$, and that $\pi_{n}(x)=\pi_{n-1}(x)\left(x-z_{n+1}\right)$ we get

$$
R_{n}\left(f ; x_{0}, x_{k}\right)=-y\left[x_{0}, z_{2}, \cdots, z_{n}, \xi, \eta\right] \sum_{i=1}^{n} C_{i k}^{n} \pi_{n}{ }^{\prime}\left(z_{i}\right) \text {. }
$$

Recalling that $y(x)=\int_{x_{0}}^{x} f(t) d t$ where $f \in C^{n}[a, b]$ gives

$$
y\left[x_{0}, z_{2}, \cdots, z_{n}, \xi, \eta\right]=\frac{1}{(n+1) !} f^{(n)}(\bar{x}),
$$

where $\min \left(x_{0}, z_{1}, \cdots, z_{n+1}\right) \leqq \bar{x} \leqq \max \left(x_{0}, z_{1}, \cdots, z_{n+1}\right)$. Thus (4.12) in conjunction with (4.4) implies the integration formula (3.2) is simplex.

In applying Theorem 4.2, since the terms (4.3) and $C_{1 k}^{n} \pi_{n-1}\left(z_{1}\right)$ must be computed anyway, the constant multiplying $-y\left[x_{0}, z_{2}, \cdots, z_{n}, \xi, \eta\right]$ in (4.4) is just the sum of $C_{1 k}^{n} \pi_{n-1}\left(z_{1}\right)$ and the terms in (4.3). This is seen to be true by $(4.10)$.

5. Examples of Closed Type Formulas. Consider as the first example Simpson's Rule

$$
\int_{a_{0}}^{a_{2}} f(t) d t \sim \frac{h}{3}\left\{f\left(a_{0}\right)+4 f\left(a_{1}\right)+f\left(a_{2}\right)\right\},
$$

where $a_{i}=a_{0}+i h$ for $i=0,1,2$. Since it is easy to check that Simpson's Rule is of order four, we know that corresponding to the formula (3.2) Simpson's Rule can be written in the form

$$
\int_{z_{1}}^{z_{4}} f(t) d t=\sum_{i=1}^{4} C_{i 4}^{4} f\left(z_{i}\right)+R_{4}\left(f ; z_{1}, z_{4}\right),
$$

where

$$
R_{4}\left(f ; z_{1}, z_{4}\right)=-\sum_{i=1}^{4} C_{i 4}^{4} \pi_{3}^{\prime}\left(z_{i}\right)\left(z_{i}-z_{5}\right) y\left[z_{1}, \cdots, z_{5}, z_{i}\right] .
$$

Here we have taken $z_{1}=x_{0}=a_{0}, z_{2}=z_{1}+h, z_{3}=z_{1}+\frac{3}{2} h, z_{4}=z_{1}+2 h$, and $z_{5}=z_{1}+\frac{7}{4} h$. Note $z_{3}<z_{5}<z_{4}$. By pairing up the $C_{i 4}^{4}$ 's with Simpson's Rule we get $C_{14}^{4}=\frac{1}{3} h, C_{24}^{4}=\frac{4}{3} h, C_{34}^{4}=0, C_{44}^{4}=h / 3$.

Since $z_{1}=x_{0}$,

$$
\pi_{3}(z)=\left(z-z_{1}\right)\left(z-z_{2}\right)\left(z-z_{3}\right)\left(z-z_{4}\right),
$$

and we have $\pi_{3}{ }^{\prime}\left(z_{1}\right)=-3 h^{3}, \pi_{3}{ }^{\prime}\left(z_{2}\right)=\frac{1}{2} h^{3}, \pi_{3}{ }^{\prime}\left(z_{3}\right)=-\frac{3}{8} h^{3}$ and $\pi_{3}{ }^{\prime}\left(z_{4}\right)=h^{3}$. We 
now see that Theorem 4.2 applies because

$$
\begin{aligned}
& \left(z_{1}-z_{2}\right) \sum_{i=1}^{1} C_{i 4}^{4} \pi_{3}{ }^{\prime}\left(z_{i}\right)=h^{5}, \\
& \left(z_{2}-z_{3}\right) \sum_{i=1}^{2} C_{i 4}^{4} \pi_{3}^{\prime}\left(z_{i}\right)=\frac{1}{6} h^{5}, \\
& \left(z_{3}-z_{4}\right) \sum_{i=1}^{3} C_{i 4}^{4} \pi_{3}^{\prime}\left(z_{i}\right)=\frac{1}{6} h^{5}, \\
& \left(z_{4}-z_{5}\right) \sum_{i=1}^{4} C_{i 4}^{4} \pi_{3}^{\prime}\left(z_{i}\right)=0,
\end{aligned}
$$

and $C_{14}^{4} \pi_{3}\left(z_{1}\right)=0$. Hence for Simpson's Rule

$$
R_{4}\left(f ; z_{0}, z_{4}\right)=-y\left[z_{1}, z_{2}, z_{3}, z_{4}, \xi, \eta\right] \frac{4}{3} h^{5},
$$

where $z_{1} \leqq \xi, \eta \leqq z_{1}+2 h$. If we use the fact that $y\left[z_{1}, z_{2}, z_{3}, z_{4}, \xi, \eta\right]=$ $(1 / 5 !) f^{(4)}(\bar{x})$ for $z_{1} \leqq \bar{x} \leqq z_{1}+2 h$ we get

$$
R_{3}\left(f ; z_{0}, z_{4}\right)=-\frac{h^{5}}{90} f^{(4)}(\bar{x}) .
$$

Note that since $\sum_{i=1}^{4} C_{i 4}^{4} \pi_{3}{ }^{\prime}\left(z_{i}\right)=0$ it does not matter what values $z_{5}$ has, because the only place $z_{5}$ enters in is in the term, see (4.9),

$$
y\left[z_{1}, z_{2}, z_{3}, z_{4}, z_{4}, z_{5}\right]\left(z_{4}-z_{5}\right) \sum_{i=1}^{4} C_{i 4}^{4} \pi_{3}^{\prime}\left(z_{i}\right)
$$

and this term is zero.

For the second example we consider the general four point closed quadrature formula

$$
\int_{-1}^{1} f(x) d x \sim C_{0} f(-1)+C_{1} f(\alpha)+C_{2} f(\beta)+C_{3} f(1),
$$

where $-1<\alpha<\beta<1$. The coefficients $C_{i}$ are given by

$$
C_{i}=\int_{-1}^{1} L_{i}^{3}(s) d s
$$

where

$$
\begin{aligned}
L_{0}{ }^{3}(s) & =\frac{(s-\alpha)(s-\beta)(s-1)}{-2(1+\alpha)(1+\beta)}, \\
L_{1}{ }^{3}(s) & =\frac{(s+1)(s-\beta)(s-1)}{(\alpha+1)(\alpha-\beta)(\alpha-1)}, \\
L_{2}{ }^{3}(s) & =\frac{(s+1)(s-\alpha)(s-1)}{(\beta+1)(\beta-\alpha)(\beta-1)} \\
L_{3}{ }^{3}(s) & =\frac{(s+1)(s-\alpha)(s-\beta)}{2(1-\alpha)(1-\beta)} .
\end{aligned}
$$


Carrying out the integration in (5.8) gives

$$
\begin{aligned}
& C_{0}=-\frac{1}{2(1+\alpha)(1+\beta)}\left[-\frac{2}{3}(1+\alpha+\beta)-2 \alpha \beta\right], \\
& C_{1}=\frac{1}{(\alpha+1)(\alpha-\beta)(\alpha-1)}\left[\frac{4}{3} \beta\right], \\
& C_{2}=\frac{1}{(\beta+1)(\beta-\alpha)(\beta-1)}\left[\frac{4}{3} \alpha\right], \\
& C_{3}=\frac{1}{2(1-\alpha)(1-\beta)}\left[-\frac{2}{3}(-1+\alpha+\beta)+2 \alpha \beta\right] .
\end{aligned}
$$

From Theorem 4.2 the sufficient condition for these formulas to be simplex of order four is that

$$
\begin{aligned}
& {\left[-\frac{2}{3}(1+\alpha+\beta)-2 \alpha \beta\right]} \\
& {\left[-\frac{2}{3}(1+\alpha-\beta)-2 \alpha \beta\right]} \\
& {\left[-\frac{2}{3}(1-\alpha-\beta)-2 \alpha \beta\right]}
\end{aligned}
$$

do not change sign. The fourth term is zero and need not be considered, as is also $C_{0} \pi_{3}(-1)$.

By setting the terms in (5.11) equal to zero we can divide the triangle $-1<\alpha$, $\beta<1, \alpha<\beta$ up as shown in Figure 5.1. Excluding the lines $\alpha=\beta, \alpha=-1$, and $\beta=1$, the disjoint shaded region indicates where (a), (b), and (c) of (5.11) have the same sign. Thus any point $(\alpha, \beta)$ in this shaded region corresponds with a simplex formula $(5.7)$ of order four. The point $(-1 / \sqrt{ } 3,1 / \sqrt{ } 3)$ corresponds with the Gauss-Legendre quadrature formula for two points, which is known to be

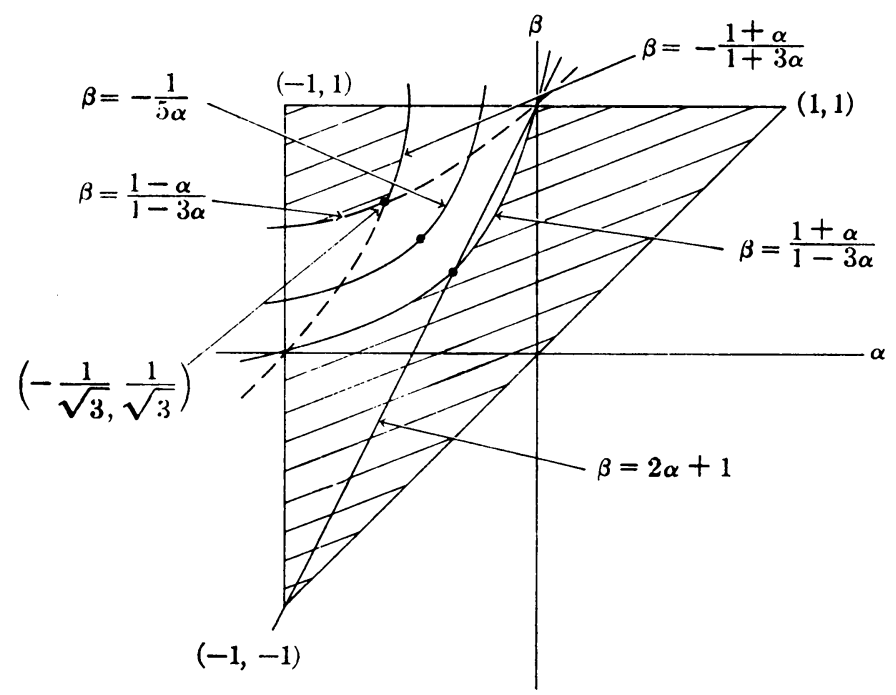

Figure 5.1 
simplex of order four. Similarly the points $(\alpha, \beta)$ in the shaded region and on the lines $\alpha=0$ or $\beta=0$ correspond with Simpson's Rule.

In [5] Bragg and Leach have an example in Section 4 which indicates that on the line $\beta=2 \alpha+1$ the formulas are simplex of order four. In Figure 5.1 this line is shown, and it is quite clear that the line enters the unshaded region. This indicates that the conditions defining the shaded region are really only sufficient conditions.

Since the $m$ th divided difference of a polynomial of degree $m$ is a constant, if the sum $\sum_{i=1}^{n} C_{i k}^{n} \pi_{n}{ }^{\prime}\left(z_{i}\right)$ in (4.4) equals zero we know the formula under consideration has order greater than or equal to $n+1$. If we solve for $\alpha$ and $\beta$ so that for formula (5.7)

$$
C_{0} \pi_{4}^{\prime}(-1)+C_{1} \pi_{4}^{\prime}(\alpha)+C_{2} \pi_{4}^{\prime}(\beta)+C_{3} \pi_{4}^{\prime}(1)=0
$$

we get the curve $\beta=-1 / 5 \alpha$. On this curve the formulas are of order five except at the point $(-1 / \sqrt{ } 5,1 / \sqrt{ } 5)$, which is known (see [8]) to be simplex of order six. ${ }^{1}$ All other points in the triangular region correspond to formulas of order four.

6. An Example for an Open Type Formula. As an example for open type formulas consider

$$
\int_{x_{0}}^{z_{5}} f(t) d t \sim \frac{5 h}{24}\left\{11 f\left(z_{1}\right)+f\left(z_{2}\right)+f\left(z_{3}\right)+11 f\left(z_{4}\right)\right\},
$$

where $z_{i}=x_{0}+i h$ for $i=1, \cdots, 5$. Hence for this example $\pi_{n-1}(x)=\pi_{3}(x)=$ $\left(x-x_{0}\right)\left(x-z_{2}\right)\left(x-z_{3}\right)\left(x-z_{4}\right)$. Also $\pi_{n}(x)=\pi_{4}(x)=\left(x-x_{0}\right)\left(x-z_{2}\right)$. $\left(x-z_{3}\right)\left(x-z_{4}\right)\left(x-z_{6}\right)$.

Thus we have $\pi_{3}{ }^{\prime}\left(z_{1}\right)=5 h^{3}, \pi_{3}^{\prime}\left(z_{2}\right)=4 h^{3}, \pi_{3}^{\prime}\left(z_{3}\right)=-3 h^{3}, \pi_{3}^{\prime}\left(z_{4}\right)=8 h^{3}$, and $\pi_{3}\left(z_{1}\right)=-3 ! h^{4}$. Also for this example $C_{14}^{4}=\frac{55}{24} h, C_{24}^{4}=\frac{5}{24} h, C_{34}^{4}=\frac{5}{24} h$, and $C_{44}^{4}=$ $\frac{55}{2} h$.

Applying Theorem 4.2 we have

$$
\begin{aligned}
\left(z_{1}-z_{2}\right) C_{14}^{4} \pi_{3}{ }^{\prime}\left(z_{1}\right) & =(-h)\left(\frac{275}{24} h^{4}\right), \\
\left(z_{2}-z_{3}\right)\left[C_{14}^{4} \pi_{3}^{\prime}\left(z_{1}\right)+C_{24}^{4} \pi_{3}^{\prime}\left(z_{2}\right)\right] & =(-h)\left(\frac{275}{24}+\frac{5}{6}\right) h^{4}, \\
\left(z_{3}-z_{4}\right)\left[C_{14}^{4} \pi_{3}^{\prime}\left(z_{1}\right)+C_{24}^{4} \pi_{3}^{\prime}\left(z_{2}\right)+C_{34}^{4} \pi_{3}^{\prime}\left(z_{3}\right)\right] & =(-h)\left(\frac{275}{24}+\frac{5}{6}-\frac{5}{8}\right) h^{4},
\end{aligned}
$$

and

$$
\left(z_{4}-z_{5}\right)\left[C_{14}^{4} \pi_{3}^{\prime}\left(z_{1}\right)+\cdots+C_{44}^{4} \pi_{3}^{\prime}\left(z_{4}\right)\right]=(-h)\left(\frac{275}{24}+\frac{5}{8}-\frac{5}{8}+\frac{55}{3}\right) h^{4} .
$$

These quantities are all seen to be negative and $C_{14}^{4} \pi_{3}\left(z_{1}\right)=-\frac{55}{4} h^{5}$ is also negative. Hence we have from Theorem 4.2 that

$$
R_{3}\left(f, x_{0}, z_{5}\right)=y\left[x_{0}, z_{2}, z_{3}, z_{4}, \xi, \eta\right]\left\{4 \frac{275}{24}+3 \frac{5}{6}-2 \frac{5}{8}+\frac{55}{3}+\frac{55}{4}\right\} h^{5}
$$

or

$$
R_{3}\left(f, x_{0}, x_{5}\right)=\frac{475}{6} h^{5} y\left[x_{0}, z_{2}, z_{3}, z_{4}, \xi, \eta\right] .
$$

${ }^{1}$ I am indebted to the referee for pointing out the existence of this curve. 
Using now the fact that $y\left[x_{0}, z_{2}, z_{3}, z_{4}, \xi, \eta\right]=f^{(4)}(\bar{x}) / 5$ ! for $x_{0} \leqq \bar{x} \leqq z_{5}$ we have

$$
R_{3}\left(f, x_{0}, x_{5}\right)=\frac{95}{144} h^{5} f^{(4)}(\bar{x}) \text {. }
$$

Michigan State University

East Lansing, Michigan

1. F. B. Hildebrand, Introduction to Numerical Analysis, McGraw-Hill, New York, 1956. MR 17, 788.

2. W. E. Minne, Numerical Calculus Approximations, Interpolations, Finite differences, Numerical Integration, and Curve Fitting, Princeton Univ. Press, Princeton, N. J., 1949. MR $10,483$.

3. J. F. Steffenson, Interpolation, Williams and Wilkins, Baltimore, Md., 1927.

4. A. WALTER, "Zur numerischen integration," Skand. Aktuarietidskr., 1925.

5. L. R. BRAGG \& E. B. LeACH, "The remainder terms in numerical integration formulas," Amer. Math. Monthly, v. 70, 1963, pp. 70-76. MR 26 *4481.

6. V. I. KRYLOv, Approximate Calculation of Integrals, Fizmatgiz, Moscow, 1959; English transl., Macmillan, New York, 1962. MR 22 *2002; MR 26 *2008.

7. K. S. Kunz, Numerical Analysis, McGraw-Hill, New York, 1957. MR 19, 460.

8. P. J. DANIELL, "Remainders in interpolation and quadrature formulae," Math. Gaz., v. 24, 1940, pp. 238-244. MR 2, 196. 